\title{
RETRACTED ARTICLE: Rhamnolipid biosurfactants: functional properties and potential contributions for bioremediation
}

\author{
Yanling Ma $\cdot$ Yanpeng Li $\cdot$ Chao Huang $\cdot$ Yuexin Tian $\cdot$ Zhidan Hao
}

Received: 23 May 2018/Accepted: 17 October 2018/Published online: 24 October 2018

(C) Springer Nature B.V. 2018

The Editor in Chief has retracted this article (1) because it contains sections that substantially overlap with the following articles (amongst others) $(2,3)$. All authors agree to this retraction.

1) Yanling Ma, Yanpeng Li, Chao Huang, Yuexin Tian, Zhidan Hao, Rhamnolipid biosurfactants: functional properties and potential contributions for bioremediation, Biodegradation, 2018, pp 1-11, https://doi.org/10.1007/s10532-018-9862-9

2) Zhuotong Zeng, Yang Liu, Hua Zhong, Rong Xiao, Guangming Zeng, Zhifeng Liu, Min Cheng, Cui Lai, Chen Zhang, Guansheng Liu, Lei Qin, Mechanisms for rhamnolipids-mediated biodegradation of hydrophobic organic compounds, Science of The Total Environment, Volume 634, 1 September 2018, Pages 1-11, https://doi.org/10.1016/j.scitotenv.2018.03.349

3) Binbin Shao, Zhifeng Liua, Hua Zhong, Guangming Zeng, Guansheng Liu, Mingda Yu, Yang Liu, Xin Yang, Zhigang Li, Zhendong Fang, Juntao Zhang, Chenghao Zhao, Effects of rhamnolipids on microorganism characteristics and applications in composting: A review, Microbiological Research, Volume 200, July 2017, Pages 33-44, https://doi.org/ 10.1016/j.micres.2017.04.005

Electronic supplementary material The online version of this article (https://doi.org/10.1007/s10532-018-9862-9) contains supplementary material, which is available to authorized users.

Y. Ma $(\bowtie) \cdot$ Y. Li · C. Huang · Y. Tian · Z. Hao Shaanxi Provincial Key Laboratory of Biotechnology, Key Laboratory of Resources Biology and Biotechnology in Western China, Ministry of Education, College of Life Science, Northwest University, 229 Taibai North Rd, Xi'an 710069, Shaanxi, China e-mail: mayanling@nwu.edu.cn 